5-1-1989

\title{
An Alternative Approach to the Teaching of Rotational Dynamics
}

James A. Lock

Cleveland State University, j.lock@csuohio.edu

Follow this and additional works at: https://engagedscholarship.csuohio.edu/sciphysics_facpub

Part of the Physics Commons

How does access to this work benefit you? Let us know!

\section{Publisher's Statement}

Copyright 1989 American Association of Physics Teachers. The article appeared in American Journal of Physics 57 (1989): 428-432 and may be found at http://aapt.scitation.org/doi/ 10.1119/1.15996

\section{Original Citation}

Lock, James A. "An Alternative Approach to the Teaching of Rotational Dynamics." American Journal of Physics 57 (1989): 428-432.

\section{Repository Citation}

Lock, James A., "An Alternative Approach to the Teaching of Rotational Dynamics" (1989). Physics Faculty Publications. 82.

https://engagedscholarship.csuohio.edu/sciphysics_facpub/82

This Article is brought to you for free and open access by the Physics Department at EngagedScholarship@CSU. It has been accepted for inclusion in Physics Faculty Publications by an authorized administrator of EngagedScholarship@CSU. For more information, please contact library.es@csuohio.edu. 


\title{
An alternative approach to the teaching of rotational dynamics
}

James A. Lock

\begin{abstract}
An alternative approach to the stability of torque-free rotations and to top nutation that emphasizes conservation laws and physical reasoning is examined. It is felt that such an approach, which is largely free of sophisticated mathematics, would be useful in teaching these topics in rotational motion at the introductory physics level.
\end{abstract}

\section{INTRODUCTION}

At the noncalculus physics and freshman engineering physics levels, the subject of the dynamics of tops either is not treated at all, or only a derivation of the steady-state slow precessional frequency is given.' It is not until the junior-senior physics major course or mechanical engineering major course in advanced mechanics that topics such as rotational stability and the nutation of tops are analyzed. Since children observe the motion of toy tops and watch the rotation of blocks thrown in the air, at a relatively early age they can develop an amazement and curiosity as to why spinning objects behave in the strange ways that they do. In light of this, it is regrettable that the discussion of these curious rotational motions is delayed so long in the scientific education of the college student

When such topics are eventually treated in advanced me. chanics textbooks, they are usually examined in terms of the solutions of the Euler equations, the set of nonlinear coupled differential equations that describe rotational motion. The mathematical analysis of these equations explains in great detail how the unusual motion of tops comes about. But at the end of going through the mathematics, I remain secretly uncomfortable, feeling that I still have not come any closer to understanding physically why the top moves
as it does.

This article is an attempt to answer the question physically of why rotating objects behave as they do in two easily observable situations. These are the stability of torque-free rotations and top nutation. The analyses require no differential equations or calculus. Rather, physical reasoning is emphasized and the only mathematics used is algebra and trigonometry. It is hoped that this approach can put the analysis of advanced but interesting rotational problems within the scope of a noncalculus-based physics course

\section{THE STABILITY OF TORQUE-FREE ROTATIONS}

We consider a parallelepiped block whose symmetry axes passing through the center of mass are labeled 1, 2 , and 3 as in Fig. 1. The moments of inertia about these axes are $I_{1}, I_{2}$, and $I_{3}$, respectively, and are assumed to be un. equal. When the block is spun about one of these axes and is thrown in the air, it is observed that rotations about the largest and smallest moment of inertia symmetry axis are stable but that rotations about the intermediate moment of inertia symmetry axis are unstable. ${ }^{2}$ We wish to understand why this stability or instability occurs.

When the block is spinning in the air, gravity acts through its center of mass and produces zero torque as measured by observers whose origins are at the center of mass. As a result, such observers see the angular momentum vector as being conserved. Since there are no dissipative forces, rotational kinetic energy is conserved as well. We consider two observers who describe the rotational motion of the block. The first observer $A$ is an inertial observer who keeps track of the block's spatial orientation. The $x, y$, and $z$ axes of a coordinate system parallel to A's coordinate axes and passing through the center of mass of the block are shown in Fig. 1. The 3-symmetry axis of the block has the direction indicated by the spherical coordinate system angles $\theta$ and $\phi$. Once the 3-symmetry axis points in this direction, the orientation of the cross-sectional plane of the block containing the 1-and 2-symmetry axes is given by the 


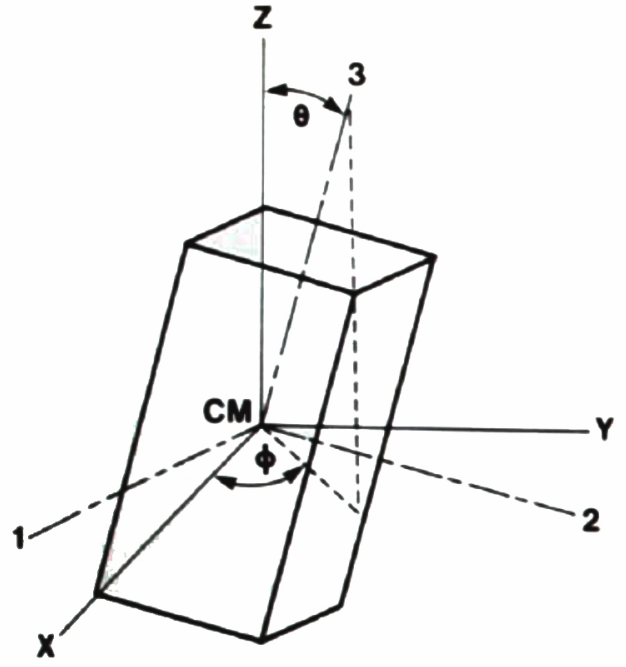

Fig. 1. A block with the symmetry axes, 1, 2, and 3 and the $x, y$, and $z$ axes of an inertial observer. The 3 -axis makes an angle $\theta$ with the $z$ axis and its projection in the $x y$ plane makes an angle $\phi$ with the $x$ axis.

angle $\psi$. The second observer P keeps track of the block's spinning. $P$ rides along on the block with his rectangular coordinate system axes nailed on top of the block's symmetry axes. He describes the spinning of the block in terms of the rotational velocities $\omega_{1}, \omega_{2}$, and $\omega_{3}$ about the symmetry axes.

The initial conditions of the block's motion consist of specifying both its initial orientation and its initial spin. The block is taken to be initially spinning with the angular velocity $\omega_{3}(0)$ about the 3-symmetry axis, which is oriented along the $z$ direction of observer $A$ as in Fig. 2. Therefore, observer $\mathbf{A}$ sees the angular momentum vector as being

$$
L=I_{3} \omega_{3}(0) \hat{u}_{z}
$$

and the rotational kinetic energy as being

$$
\mathrm{KE}=\frac{1}{2} I_{3} \omega_{3}^{2}(0) \text {. }
$$

We call this the $\left[\theta=0^{\circ}, \omega_{3}(0)\right]$ configuration of the block. There is another configuration of the block, which possesses exactly the same angular momentum vector and the

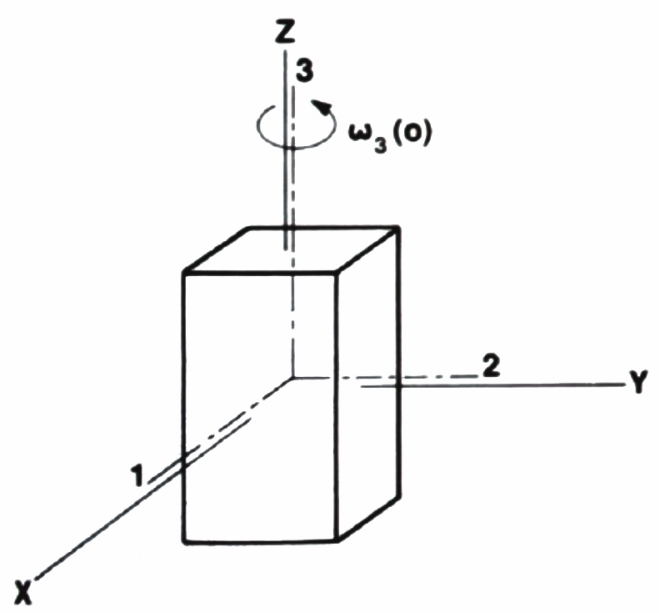

Fig. 2. The initial conditions of the block. It spins with the angular velocity $\omega_{3}(0)$ about the 3-axis, which is in the $z$ direction.

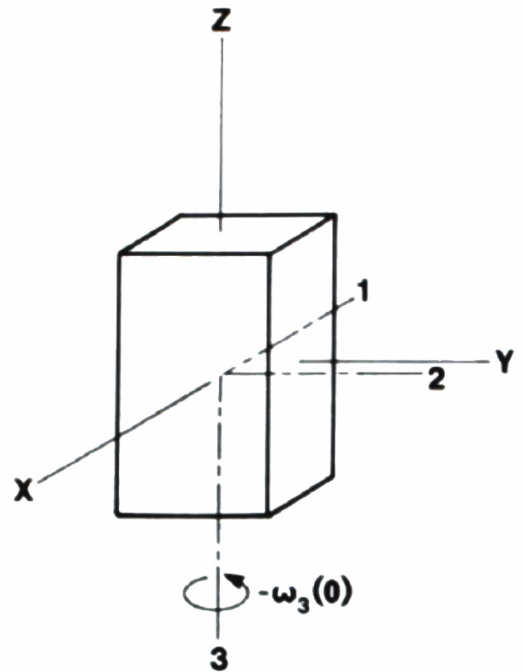

Fig. 3. An equivalent configuration of the block that has the same angular momentum vector and rotational kinetic energy. Its orientation and its spinning about the 3 -axis have been reversed.

same rotational kinetic energy. This is when the block's orientation is reversed and its spinning with respect to the 3-symmetry axis is reversed as in Fig. 3. We call this the $\left[\theta=180^{\circ},-\omega_{3}(0)\right]$ configuration. Since both configurations are totally equivalent, when the block is spun and thrown in the air, are the initial conditions enough to specify which one of them will be dominant in the resulting motion?

This question is answered in terms of the orientation paths available to the block. A path is analogous to the frames of a movie that starts with the block in the $\left[\theta=0^{\circ}\right.$, $\omega_{3}(0)$ ] configuration and shows it turning until it is finally in the $\left[\theta=180^{\circ},-\omega_{3}(0)\right]$ configuration. If a path between these two configurations can be found that conserves the angular momentum vector and the rotational kinetic energy at every step along the way, then the initial conditions are not enough to specify which of the equivalent configurations dominates the motion. Rather, the block traverses the path of orientations back and forth, oscillating between the two. This represents an unstable rotation. On the other hand, if there is no path between the two that conserves the angular momentum vector and the rotational kinetic energy at every step along the way, the initial conditions are compatible with only one of the configurations. The turning of the block to the other configuration is forbidden. This represents a stable rotation.

There are many paths between the two configurations for which the angular momentum vector is conserved. If the block is at some moment in the $(\theta, \phi, \psi)$ orientation and its spinning is given by

$$
\begin{aligned}
& \omega_{1}=-\left(I_{3} / I_{1}\right) \omega_{3}(0) \sin \theta \cos \psi, \\
& \omega_{2}=\left(I_{3} / I_{2}\right) \omega_{3}(0) \sin \theta \sin \psi, \\
& \omega_{3}=\omega_{3}(0) \cos \theta,
\end{aligned}
$$

then the angular momentum vector seen by observer $A$ is given by Eq. (1). However, it is difficult to make any of these paths conserve the rotational kinetic energy

$$
\mathrm{KE}=\frac{1}{2} I_{1} \omega_{1}^{2}+\frac{1}{2} I_{2} \omega_{2}^{2}+\frac{1}{2} I_{3} \omega_{3}^{2}
$$

at every step along the way as well. At the point on the path specified by Eq. (3), conservation of the rotational kinetic 


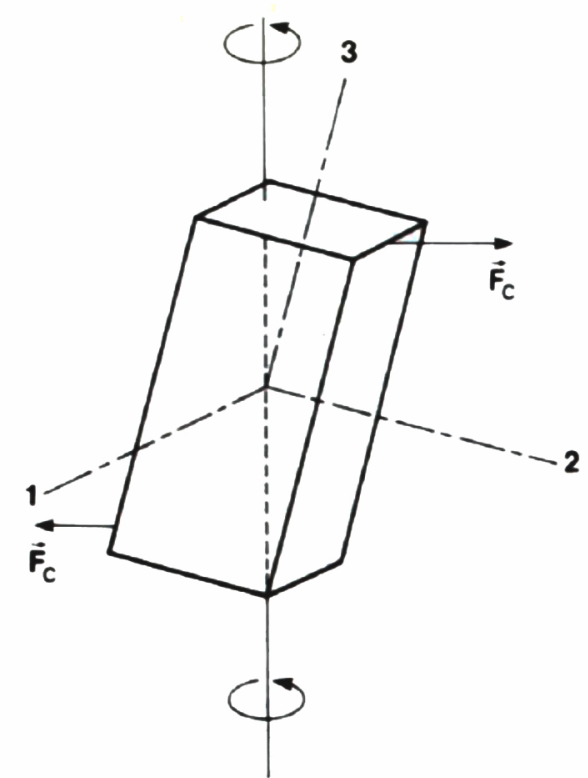

Fig. 4. The initial spinning of the block approximately about the $z$ axis. $\mathbf{F}$. is the centrifugal force acting on the asymmetric mass concentrations.

energy requires that Eq. (3) substituted into Eq. (4) must be equal to the initial kinetic energy of $\mathrm{Eq} .(2)$. When this is done, the $\theta$ dependence cancels out and one is left with

$$
\sin ^{2} \psi=I_{2}\left(I_{1}-I_{3}\right) / I_{3}\left(I_{1}-I_{2}\right) \text {. }
$$

The condition that $0 \leqslant \sin ^{2} \psi \leqslant 1$ further requires that either $I_{1}>I_{3}>I_{2}$ or $I_{2}>I_{3}>I_{1}$. Thus only when $I_{3}$ is the intermediate moment of inertia can one find a path of orientations between the two equivalent configurations that conserves both energy and angular momentum. When $I_{3}$ is the intermediate moment of inertia, what gives the block the initial push to start it along the path between the two equivalent configurations? The answer is found in the fact that when one throws the block in the air, it is difficult to release it so that it spins exactly about the 3 -axis. ${ }^{2}$ It is more likely that the released block will end up spinning approximately about the 3-axis as shown in Fig. 4. Since the block is slightly asymmetric in its initial spinning, the extra concentrations of mass with respect to the spin axis in the upper right and lower left of Fig. 4 will be pulled perpendicular to the spin axis by centrifugal force. This force is denoted by $\mathbf{F}_{c}$ in Fig. 4 and it causes an additional rotation of the block about the 1-axis. The combination of the rotation of the block about the spin axis and the rotation about the 1 -axis produced by centrifugal force starts the block moving along the path of allowed orientations oscillating between the two equivalent configurations.

\section{TOP NUTATION}

We consider a symmetric top with the base point fixed and whose center of mass is a distance $h$ from the base point. The moments of inertia with respect to the 1-,2-, and 3 -axes of Fig. 5 are $I_{1}=I_{2}=I_{12}$ and $I_{3}$. The top is spun with the speed $\omega_{3}(0)$ about the 3-symmetry axis. If the top is given a small tangential push as it is released, it is observed that it does not fall due to gravity. Instead, it precesses uniformly. If the top is released without the small tangential push, it is observed that for a short time its cen-

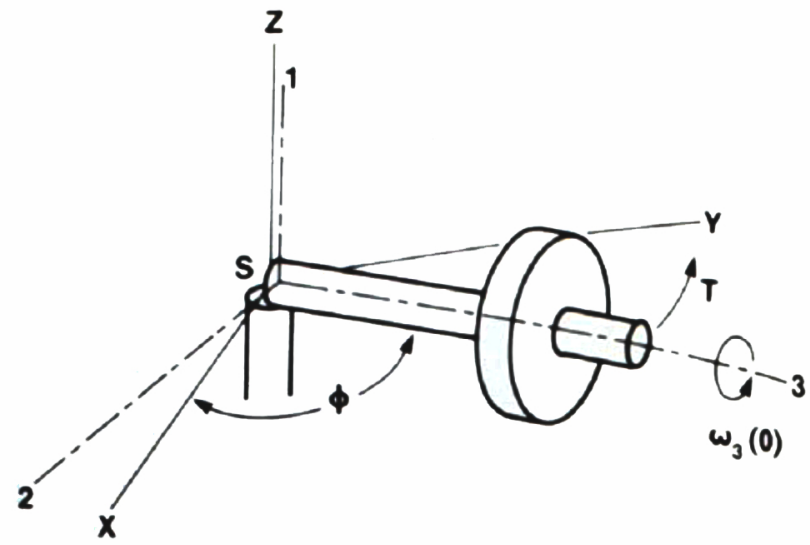

Fig. 5. The initial conditions of the top. It spins with the angular velocity $\omega_{1}(0)$ about the 3-axis, which is in the xy plane of an incrtial observer The base support $S$ is fixed and the tangential direction $T$ is the direction of precession.

ter of mass does fall due to gravity. But then the center of mass reaches a low point and the top bounces back upwards as if it were bouncing off of something that we cannot see. This vertical bouncing of the center of mass is called nutation. We wish to understand why the nutation bouncing occurs and what the top seems to be bouncing off.

Initially the top is oriented with its 3-symmetry axis in the $x y$ plane of observer $\mathbf{A}$ whose origin is now at the base of the top as in Fig. 5. Gravity again acts through the top's center of mass and produces a torque in the $x y$ plane perpendicular to the 3-axis. This perpendicular or tangential direction $T$ is shown in Fig. 5. Since the gravitational torque points in the $T$ direction, the angular momentum in that direction changes and gives rise to the precession of the top. 'The reaction forces at $S$ produce no torque on the top with respect to the coordinate system whose origin is at $S$. Thus the gravitational torque is the only torque in the problem and the portion of the angular momentum vector perpendicular to $T$ is conserved. In this example, this perpendicular portion is the plane containing the $z$ axis and the 3-symmetry axis. These conserved components of the an-

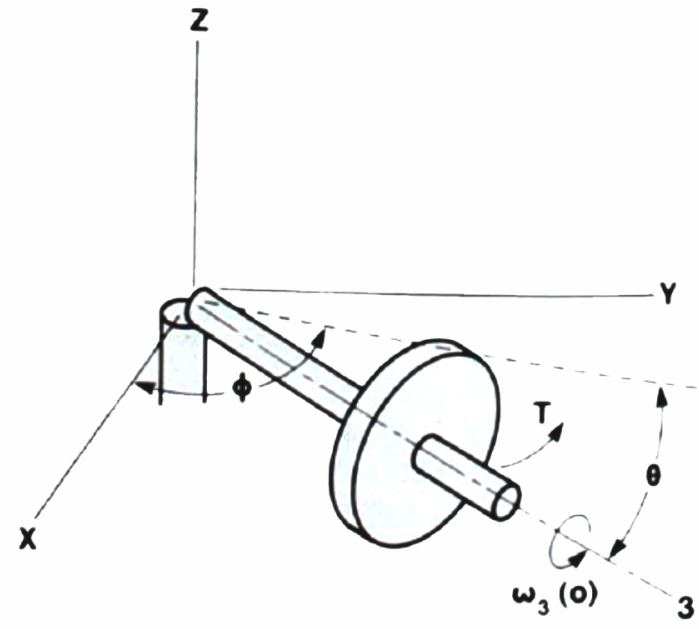

Fig. 6. The fallen configuration of the top. The 3 -axis is an angle $\theta$ below the $x y$ plane and the top precesses in the tangential direction $I$ with the angular velocity $\omega_{0}$ 


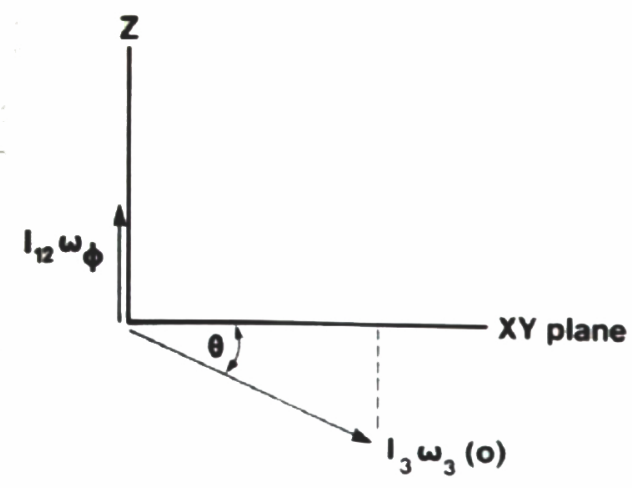

Fig. 7. The angular momenta of spinning and precession when the top is in the fallen configuration. Conservation of angular momentum requires that $L_{:}=0$.

gular momentum are then

$$
\begin{aligned}
& L_{3}=I_{3} \omega_{3}(0), \\
& L_{z}=0,
\end{aligned}
$$

as seen by observer A. Since gravity initially causes the top's center of mass to fall, a path of orientations between the initial configuration of Fig. 5 and the fallen configuration of Fig. 6 must exist, which conserves $L_{3}, L_{2}$, and energy at every step along the way. It should be noted in Fig. 6 that as a matter of convenience the angle $\theta$ is now taken to be the angle of the 3-symmetry axis below the $x y$ plane

Again, it is easy to find a path that conserves $L$. In Fig. 6 , in order to balance the downward component of the angular momentum due to spinning, $I_{3} \omega_{3}(0) \sin \theta$, the top must precess in the tangential direction with the angular speed $\omega_{\phi}$ generating an upward angular momentum. $I_{12} \omega_{\phi}$. This is shown in Fig. 7. Conservation of $L_{z}$ then requires that

$$
\omega_{\phi}=\left(I_{3} / I_{12}\right) \omega_{3}(0) \sin \theta \text {. }
$$

In order to conserve $L_{3}$ as well, the top must increase slightly its spinning speed. However, this increase is proportional to $\sin ^{2} \theta$, which is small and can be ignored since we assume that $\theta$ remains near $0^{\circ}$. If $\omega_{\theta}$ is the angular velocity of the falling of the top in the direction of increasing $\theta$, then the initial energy is

$$
E=\frac{1}{2} I_{3} \omega_{3}^{2}(0)
$$

and the energy in the fallen configuration is

$$
\boldsymbol{E}=\frac{1}{2} I_{3} \omega_{3}^{2}(0)+\frac{1}{2} I_{12} \omega_{\phi}^{2}+\frac{1}{2} I_{12} \omega_{\theta}^{2}-m g h \sin \theta .
$$

Simultaneous conservation of energy and $L_{z}$ for the path requires that

$$
I_{12} \omega_{\theta}^{2}=m g h \sin \theta-\frac{1}{2}\left(I_{3}^{2} / I_{12}\right) \omega_{3}^{2}(0) \sin ^{2} \theta .
$$

Equations (9) and (10) have two interesting physical interpretations. The first one deals with why falling motion of the top ceases. As the top falls, its potential energy is converted into kinetic energy. The kinetic energy is of two types; the kinetic energy of falling, $\frac{1}{2} I_{12} \omega_{\theta}^{2}$, and the kinetic energy of precession, $\frac{1}{2} I_{12} \omega_{\phi}^{2}$. The decrease in potential energy goes as $\sin \theta$ but the increase in precessional kinetic energy goes as $\sin ^{2} \theta$ in order to conserve $L_{z}$. For small $\theta$, $\sin ^{2} \theta$ increases much slower than $\sin \theta$ and so the difference between the potential energy loss and the precessional kinetic energy gain goes into the kinetic energy of falling. However, for somewhat larger $\theta, \sin ^{2} \theta$ increases faster than $\sin \theta$, the precessional kinetic energy claims all the gravitational potential energy that is released, and nothing is left over for the kinetic energy of falling. As a result, when

$$
\sin \theta=2 m g h I_{12} / I_{3}^{2} \omega_{3}^{2}(0),
$$

the lowest point in the falling is reached. Below this angle, the kinetic energy of the falling motion cannot obtain any of the potential energy loss in order that the falling continue.

The second interpretation of Eqs. (9) and (10) deals with why the top bounces upward when the low point of Eq. ( 11 ) is reached. For small angles $\theta$, the behavior of the top in the $\theta$ direction is as if it were subject to the potential energy

$$
U=-m g h \sin \theta+\frac{1}{2}\left(I_{3}^{2} \omega_{3}^{2}(0) / I_{12}\right) \theta^{2} .
$$

This potential energy may be interpreted as arising from the torque due to gravity and the torque produced by an effective torsional spring whose stiffness constant is

$$
K=I_{3}^{2} \omega_{3}^{2}(0) / I_{12}
$$

and which always tries to restore the orientation of the top to its initial conditon of $\theta=0^{\circ}$. In a number of situations in mechanics, it is useful to speak of fictitious forces or pseudoforces such as centrifugal force or the Coriolis force. An inertial observer does not consider these entities as true forces. Rather, he considers them to be part of the acceleration of the object. However, to a noninertial observer riding along with the object, these entities feel like forces and are compensated for by various passive forces such as tension or reaction forces. The tendency of the top to try to maintain its initial conditions can be interpreted as another fictitious force. To an inertial observer, $\frac{1}{2} K \theta^{2}$ is really the precessional kinetic energy. But, to a noninertial observer riding along with the precession and watching only the falling motion of the top, it would appear that the top uses the spring pseudotorque to attempt to recover its initial orientation when gravity has pulled the 3 -axis lower than $\theta=0^{\circ}$. The faster the top is spinning, the larger $K$ becomes, and the larger the pseudotorque of initial conditions maintenance becomes. ${ }^{\circ}$ This explains the rebound of the top from its low point. The effective spring torque is pulling the top up try-

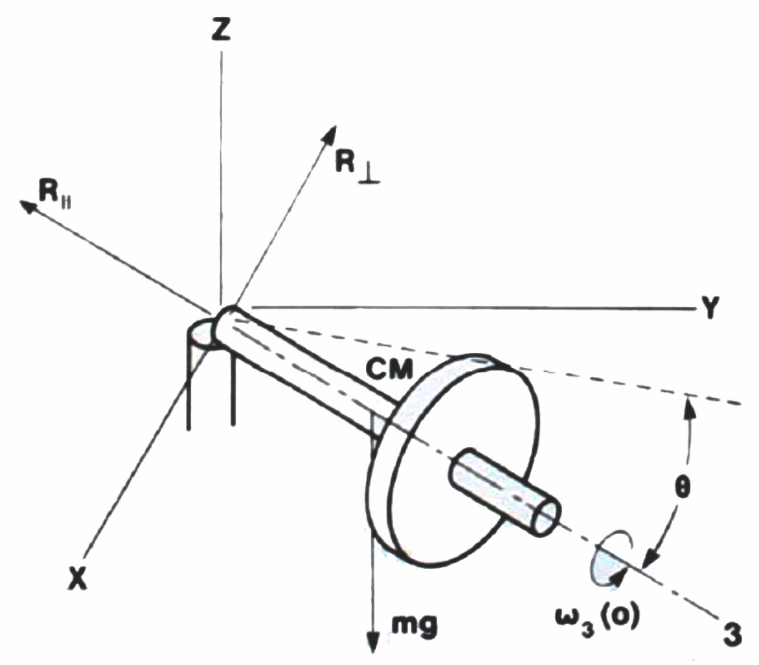

Fig. 8 . The force of gravity and the reaction forces $R_{1}$ and $R_{\rceil}$provided by the support when the top is in the fallen configuration. 
ing to recover its initial orientation just as a mass hung vertically from a spring and released is pulled back up from its low point by the force of the extended spring.

As mentioned earlier, the effects of pseudoforces are observed in the reaction forces necessary to balance them. This compensation also occurs for the springlike pseudotorque of Eq. (12). Figure 8 shows the top in its fallen orientation and indicates the reaction forces $R_{\perp}$ and $R_{\|}$, perpendicular and parallel to the 3-symmetry axis, provided by the base support. If $\alpha_{\theta}$ is the angular acceleration of the falling motion, then the motion of the center of mass is given by

$$
m h \alpha_{\theta}=m g \cos \theta-R_{1} .
$$

However, gravity and the effective torsional spring exert the torques

$$
\tau=I_{12} \alpha_{\theta}=m g h \cos \theta-\left[I_{3}^{2} \omega_{3}^{2}(0) / I_{12}\right] \theta .
$$

Combining Eqs. (14) and (15), we obtain

$R_{1}=m g \cos \theta-\frac{m^{2} g h^{2}}{I_{12}} \cos \theta+\frac{m h}{I_{12}}\left(\frac{I_{3}^{2} \omega_{3}^{2}(0)}{I_{12}} \theta\right)$.

The first two terms of Eq. (16) correspond to the value that the reaction force would have if the top were not spinning and were merely falling over. $R_{1}$ would balance a component of gravity except for the fact that the reaction is reduced by the downward acceleration of the falling top. The last term in Eq. (16) describes the increase in $R_{1}$ due to the support having to balance also the pressure exerted on it by the restoring pseudotorque when the top has been per. turbed from its initial configuration. The effective lever arm of the increased reaction force is $I_{12} / \mathrm{mh}$.

\section{CONCLUSION}

Again, it should be emphasized that in each of these two situations no mathematics beyond trigonometry and the definition of spherical coordinates was employed. The analyses instead were based on physical intuition and the requirements of conservation laws. It is hoped that with such a point of view, many topics in advanced mechanics that deal with easily observable phenomena may be intro. duced to physics students before they reach the juniorsenior-level course in advanced mechanics. If this were the case, the advanced course could then focus on supplying the mathematical framework for phenomena that the students were already familiar with from a physical point of view.

'J. Higbie, Phys. Teach 18, 210 ( 1980 )

${ }^{2}$ J. Walker, Phys. Teach. 19, 57 (1981)

${ }^{3}$ W. Case, Am. J. Phys. 45, 1107 (1977)

${ }^{4}$ P. L. Tea, Phys. Teach. 26, 22 (1988).

'A. P. French, Newtonian Mechanics (Norton. New York, 1971). pp 691-694.

${ }^{\circ}$ Another interpretation of the restoring torque is given in $\mathrm{E}$. F. Barker, Am. J. Phys. 28, 808 (1960) and in P. M. Rinard, Am J. Phys. 34, 365 (1966).

Post-print standardized by MSL Academic Endeavors, the imprint of the Michael Schwartz Library at Cleveland State University, 2017. 